\section{Signature Analysis Applied to EDS Microanalysis}

\author{
J.W. Colby \& D. C. Ward \\ $x k$, Incorporated, Clackamas, $O R^{1}$ \\ denniscward@earthlink.net
}

Most spectroscopies (FTIR, XRD, RAMAN, MS, etc.), were developed in order to identify materials. This is accomplished by capturing the spectral "signature" of an unknown and matching it to those of a comprehensive database of reference materials. Development of these spectroscopies has included efforts to embellish the associated database. EDS evolved as a resource to provide qual/quant, and development has been to improve the accuracy of quantation. Until recently, EDS was not considered a spectroscopy to provide identification of materials.

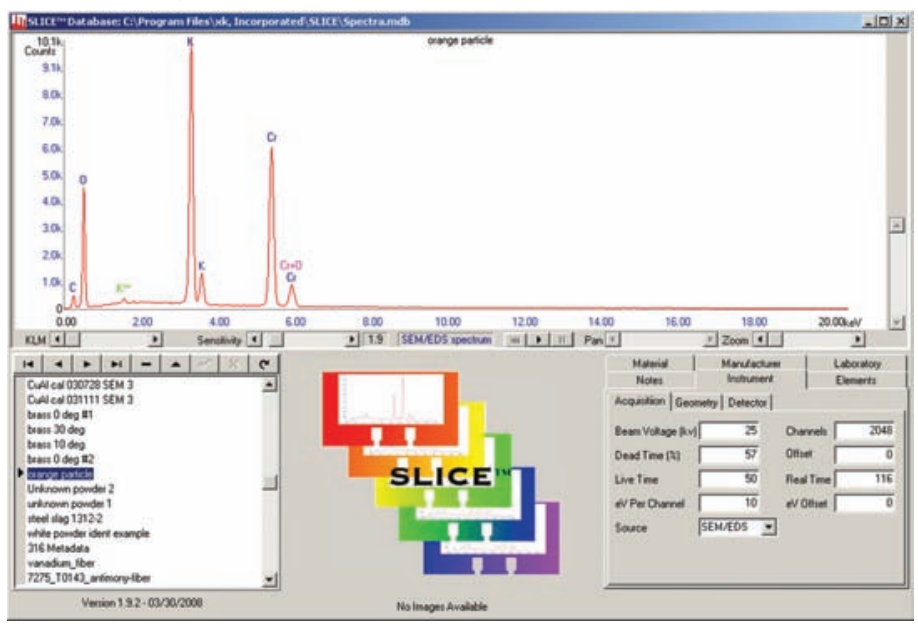

Figure 1 - Main spectral display window

In order to fully utilize SEM/EDS the Federal Bureau of Investigation evaluated the current capability to meet mission requirements for trace material characterization. It became readily apparent that a compositional approach was not sufficient, as; comparison of composition is not sufficiently discriminating, the composition of many materials is not known, and the determination of composition could not always be determined accurately for the variety of materials anticipated.

Even for the "suitable" sample, the difficulty in selecting the model and parameters for quantitation can be demonstrated in a computer program developed and patented by C. E. Fiori and C. R. Swyt at NIST in 1994, which allowed one to calculate a spectrum theoretically from a hypothetical sample. In use, the operator could pick any one of several models and parameters from many sources, to achieve the calculated spectrum, and as might be anticipated, the resulting spectra varied considerably. In fact, by choosing the parameters and model properly (or improperly), one could obtain nearly any result anticipated.

It appeared that a "traditional" spectroscopy approach to EDS could be a powerful addition to quantitation. Therefore, in 1998, the FBI awarded $\mathrm{xk}$, Inc. a contract to develop a relational database architecture and software to query the database. This led to the development of SLICE ${ }^{\text {ixt }}$ (Spectral

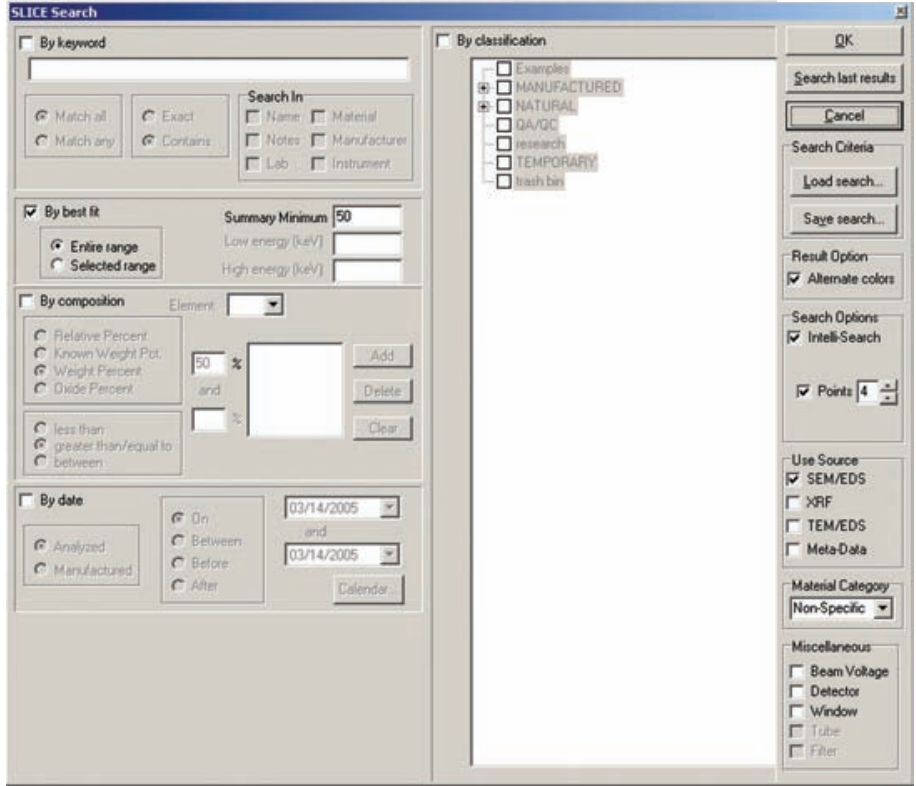

Figure 2 - Search Window for "Orange Particle"

Library for Identification and Classification Explorer). The program and database have evolved into a fully functional database program, which not only includes the spectra and chemistries, but also other metadata to facilitate and further refine the classification of spectra from unknown materials. Data mining, enhanced spectral display and comparisons, and a materials classification tree are also part of SLICE. In practice, the spectra in standard EMSA format as developed by Nestor Zaluzec, et.al. in 1991 and implemented by most EDS companies, are imported into SLICE. Chemistries may be imported if contained in the EMSA file, or may be determined within SLICE. Both peak ID and quant results may be executed within SLICE. Chemistries may be displayed as weight $\%$, atomic $\%$, relative $\%$, or in the case of minerals, clay, etc., as oxide \%. Any additional known metadata are added to the stored spectrum, including text notes, sources, physical and instrumental parameters, etc. In addition, the spectrum may be archived with SEM and optical images, $x$-ray maps, PDF documents, spreadsheets, etc, all readily retrievable. Laboratories or

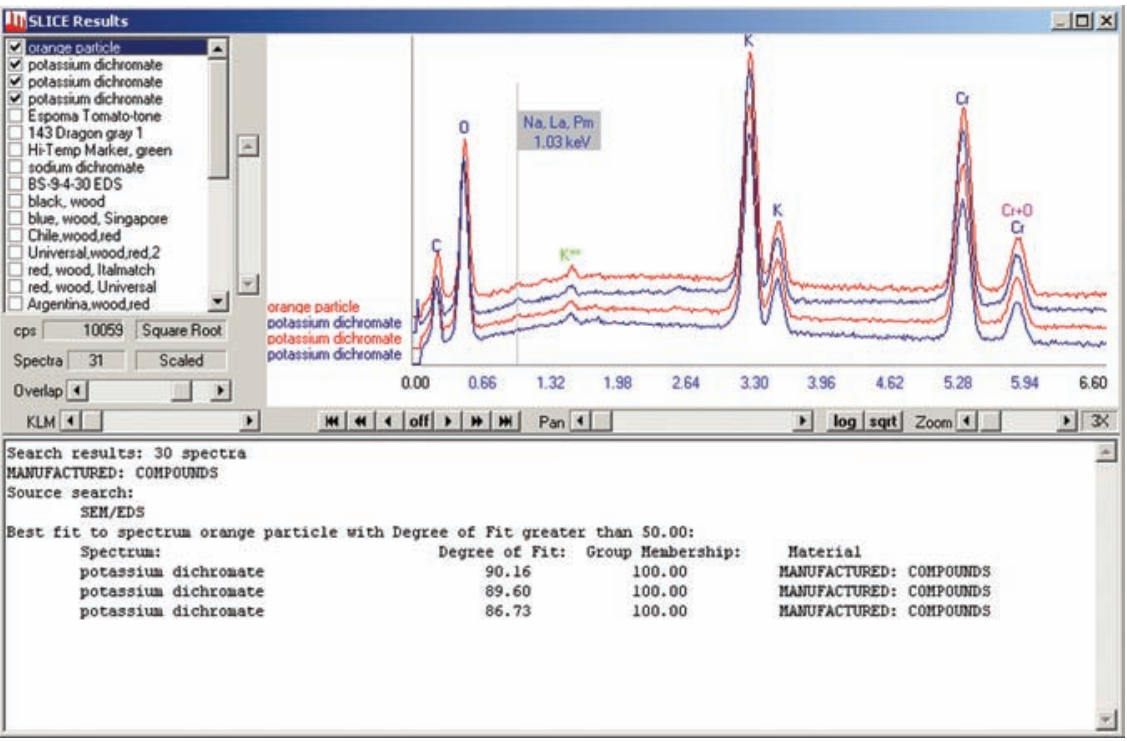

Figure 3 - Search results for the "orange particle". The spectra are displayed in the square root mode, and normalized about the cursor. 


\section{from experience... \\ learn from the}

experts.
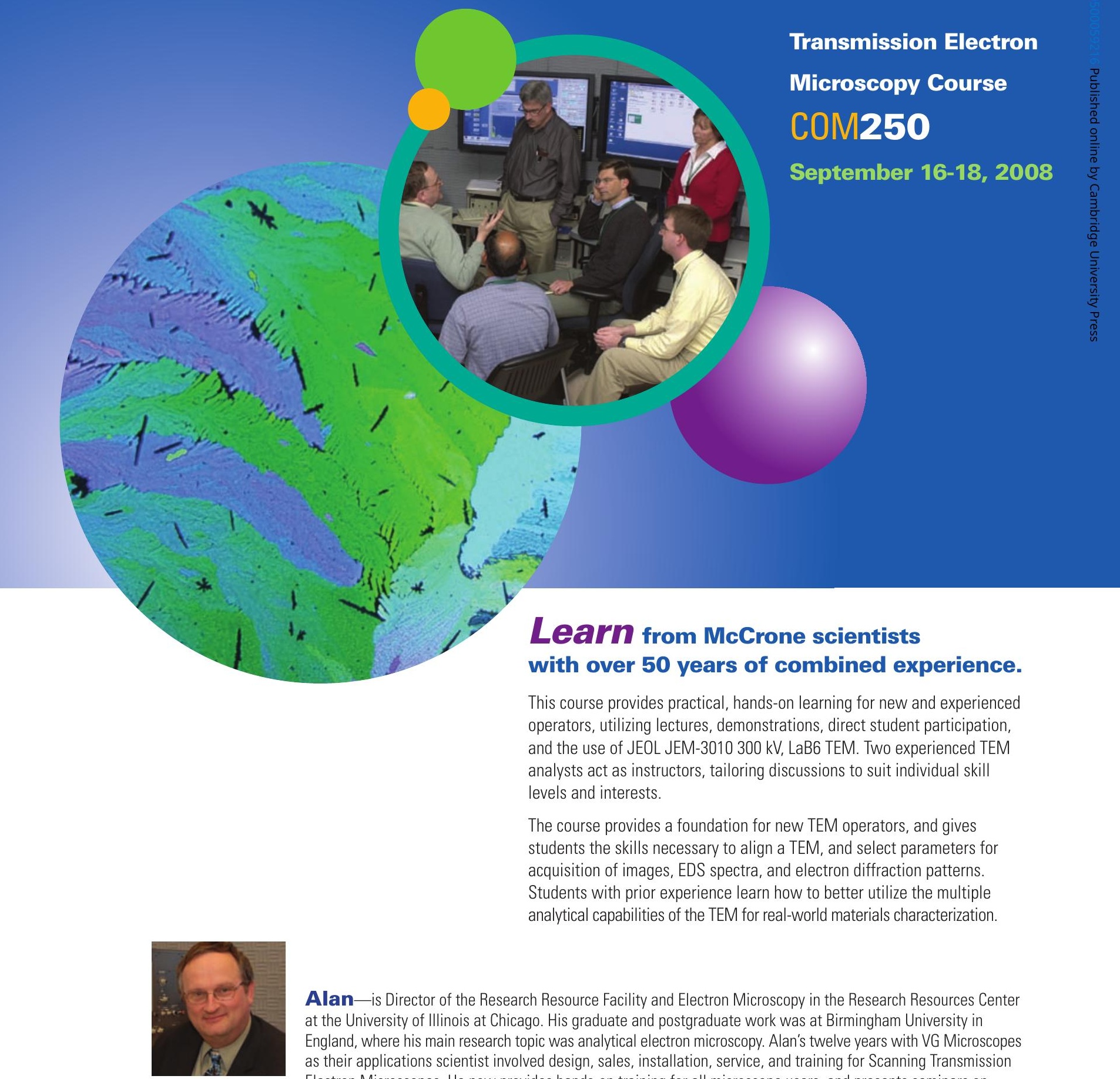

This course provides practical, hands-on learning for new and experienced operators, utilizing lectures, demonstrations, direct student participation, and the use of JEOL JEM-3010 $300 \mathrm{kV}$, LaB6 TEM. Two experienced TEM analysts act as instructors, tailoring discussions to suit individual skill levels and interests.

The course provides a foundation for new TEM operators, and gives students the skills necessary to align a TEM, and select parameters for acquisition of images, EDS spectra, and electron diffraction patterns. Students with prior experience learn how to better utilize the multiple analytical capabilities of the TEM for real-world materials characterization.

Alan-is Director of the Research Resource Facility and Electron Microscopy in the Research Resources Center at the University of Illinois at Chicago. His graduate and postgraduate work was at Birmingham University in England, where his main research topic was analytical electron microscopy. Alan's twelve years with VG Microscopes as their applications scientist involved design, sales, installation, service, and training for Scanning Transmission Electron Microscopes. He now provides hands-on training for all microscope users, and presents seminars on electron microscopy to undergraduate and graduate students.

Elaine — has been a Senior Research Scientist with McCrone Associates since 2001, applying transmission electron microscopy techniques, including high resolution imaging, electron diffraction, $\mathrm{x}$-ray microanalysis, and electron energy loss spectroscopy, to a wide variety of materials. Her consulting work for clients in industry and academia has included characterization of polymer nanocomposites, medical devices, pharmaceuticals, minerals, ceramics, and metallurgical specimens. Elaine's extensive microscopy experience includes use of dedicated STEM, TEM, and LV-SEM to study zeolites, supported metal catalysts,

filamentous carbon, and novel mixed-metal oxides.

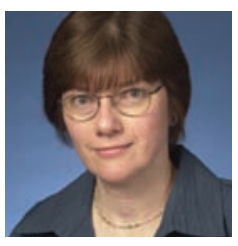




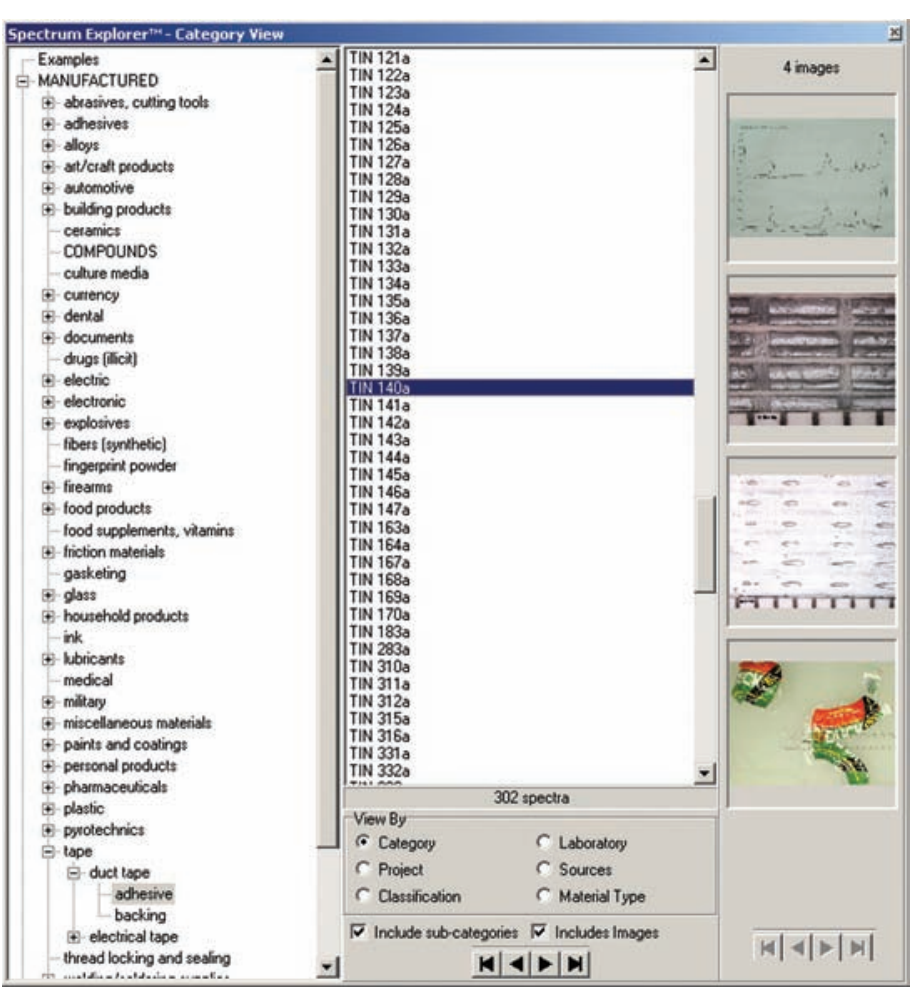

Figure 4, Spectrum Explorer, showing the classification tree on the far

left, the associated images on the right, and the list of spectra in between.

individuals may build their own database and materials classification tree (text document) to suit their particular requirements. The current database ${ }^{2}$ contains nearly 6000 spectra, from many material categories (alloys, SRM materials, minerals, commercial products, etc.). The main window of SLICE is shown in Figure 1, and consists of the spectrum from an "orange particle", and the metadata stored under the various tabs.

The notes section may contain URLs in addition to text information, and clicking the URLs will link to the appropriate web site if an external internet connection is available. Clicking the image or other documents displayed will automatically open them to full size images or documents. In this case, no images were stored with the spectrum.

Users may create their own proprietary databases in addition to the one supplied with SLICE ${ }^{2}$. In cases of failure analysis or defect revue, SLICE and the associated database become an historical resource of similar prior defect occurrences, comparable to forensic evidence relating to serial criminal activities. Since the failure analysis report (e.g. MS Word or PowerPoint document) may be associated with the spectrum as an "image", subsequent to the database query, a simple click opens the document for comparison to the current problem.

Queries can be executed based on key words (names, material, laboratory, etc., and text within the notes section), best fit, composition, date, properties, classification, instrument parameters, source, and source properties, separately or in combination with each other. As an example, from the Search Page (Figure 2), the database is searched to find likely candidates to identify/classify the "orange particle" shown in Figure 1. The best-fit algorithms have been developed and optimized to be relatively insensitive to instrumental and operating parameters, to avoid some of the problems listed above.
Spectra may be compared as the result of Search queries (Figure 3), or by direct choice from within the Spectrum Explorer shown in Figure 4.

Compared spectra can be displayed in the usual linear and $\log$ modes, and in square root mode. Overlapped spectra may be tightly or loosely overlapped, and normalized at any point in the spectra, statically or dynamically to facilitate comparison. The number of spectra overlapped can be chosen dynamically, and the metadata can be examined from any of the overlapped spectra. Data associated with the spectrum can also be tabulated.

The tabulated data can be properties specific to the material, or properties that are more general. Chemistries can also be tabulated (weight \%, atomic \%, etc.) and both chemistries and properties can be sorted in ascending or descending order. Statistics may also be displayed. The tabulated data can be copied and pasted into a document (e.g. MS Word or Excel) for further processing and/ or report generation. The tabulated chemistries for the "orange particle" and potassium dichromate spectra are shown in Figure 5 . The variations in chemistry are significant, and are due in part to the sample themselves not being flat, polished and homogeneous, as well as other uncertainties in the analysis. Nevertheless, the SLICE results strongly point to the particles being potassium dichromate. No other database entries are remotely close.

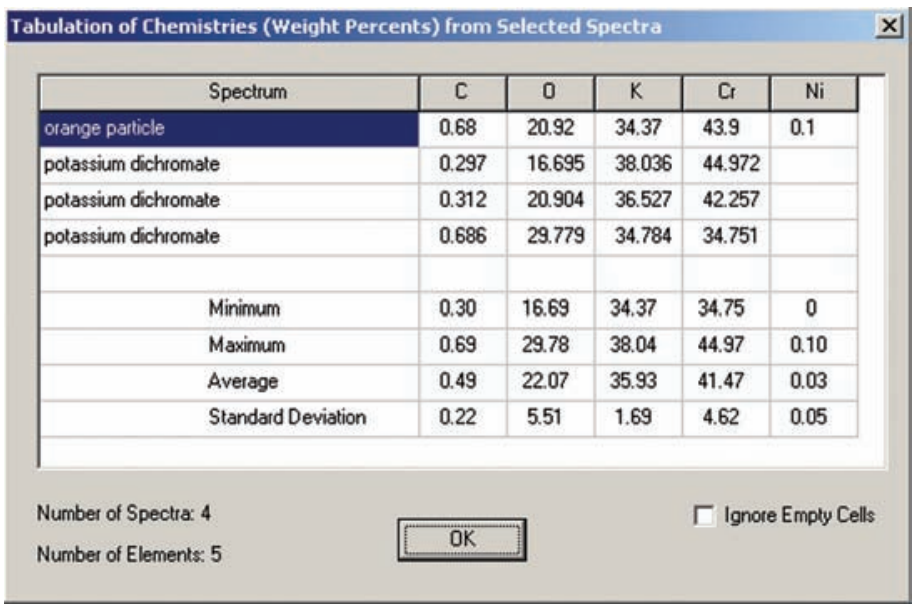

Figure 5 - Tabulated chemistries as weight percents from the "orange particle" and other potassium dichromate spectra

The majority of spectra in the current database are SEM/EDS spectra but TEM/EDS and XRF/EDS spectra are also included, and spectra of one type can be searched against spectra of another type (e.g. unknown XRF spectrum against both SEM and TEM spectra) although in general better results can be obtained against spectra of the same type.

By providing utilities for archiving and querying data, SLICE provides EDS with significantly enhanced capability, putting it on a par with other, more classical spectroscopy. Although EDS has not historically been considered a spectroscopy that provides "identification," the inclusion of this capability is significant for disciplines such as forensics, failure analysis, and process control, where material sourcing is required.

\section{References:}

1. www.xk.com

2. The original spectral database for this project was heavily populated by the FBI. The database, as subsequently modified is provided with SLICE. 


\section{Take microscopy to the world of in-situ process observation}

\section{Ever wanted to:}

see chemistry

analyze wet samples

see nanoparticle growth

allow gases to interact with your sample

see a catalyst particle function

work at elevated pressures and temperatures

...and still see atoms?

\section{or}

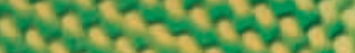

